\title{
HIT Conformance Testing: Advancing Syndromic Surveillance System Interoperability
}

\author{
Robert Snelick and Sheryl L. Taylor* \\ National Institute of Standards and Technology, Gaithersburg, MD, USA
}

\section{Objective}

Describe how the 2015 Edition of the National Institute of Standards and Technology's (NIST) Syndromic Surveillance Messaging Validation Suite continues to support federal efforts to increase healthcare information technology interoperability for timelier public health surveillance in the US; and show how this tool is used to validate messages.

\section{Introduction}

Speed, reliability, and uniformity of data collection enable syndromic surveillance (SyS) systems to provide public health authorities (PHAs) with timely information about community health threats and trends. Increasingly, healthcare information technology (HIT) is being used to accelerate and automate data collection for more real-time surveillance, reducing irregularity in how SyS data are packaged and sent by healthcare providers. Continuing to focus on patient and population health outcomes, the on-going US federal program that certifies HIT to promote interoperability has mandated broader use of an updated standard for communication of SyS data. Under the Edition 2015 federal rule tied to Medicare and Medicaid reimbursement, hospitals, in addition to emergency departments and urgent care centers, are now required to provide SyS data to PHAs using HL7 2.5.1 messages that are in conformance with Release 2.0 of the CDC's Public Health Information Network (PHIN) guide for SyS. To facilitate the intended application of this updated standard, a new version of conformance testing tools is being published, which will enable HIT developers to increase their probability of meeting the requirements outlined in the standard and lead to enhanced product interoperability and reliability.

\section{Methods}

To advance conformance to the standard and promote interoperability of HIT, the Office of the National Coordinator for Health Information Technology (ONC) has continued to maintain a voluntary HIT certification program. ONC certification testing provides the means to evaluate SyS data messages created by HIT against a mandated standard through use of a context-based validation method composed of a suite of publicly accessible, web-based tools developed by subject matter experts from NIST, ISDS, and the CDC. The updated Edition 2015 SyS Messaging Validation Suite (SySMVS) will support testing SyS data messages based on Release 2.0 of the PHIN Guide and includes test stories that set the clinical context, test SyS data, testing guidance, automated SyS message validation, and a forum for testers to ask questions or provide feedback about the suite. ONC-Accredited Testing Laboratories (ATLs) will use the suite to test HIT modules for certification, and HIT vendors can use them while developing their products. Healthcare providers and PHAs can download a version of the validation suite and use it locally to verify conformance of SyS messages containing real patient data for implementing SyS data exchange.

\section{Results}

As of August, 2015, the updated version of the Sys-MVS is being developed in anticipation of the publication of the ONC Edition 2015 HIT certification Final Rule due in Q3 2015. Several HIT vendors have been recruited to pilot test the draft SyS-MVS prior to release of the final version in December 2015. The ATLs are to begin using the SyS-MVS in January 2016, and local PHAs are anticipated to begin using the validation suite as part of their on-boarding process with their healthcare providers at that time. Feedback about the 2014 Edition of these tools was positive, indicating that the users: (1) were motivated to think through the steps for capturing/reporting syndromic data in more detail than before; (2) appreciated the subject matter expertise that generated the tools; and (3) used these tools locally to promote collection and reporting of standardized SyS data in a timely manner.

\section{Conclusions}

The 2015 Edition SyS-MVS, to be used nationally by the ATLs to test HIT for certification and locally by healthcare providers to validate electronic messages before sending their SyS data to PHAs, will help move the industry further toward true interoperability for efficient reporting and use of syndrome-based public health surveillance information.

\section{Keywords}

certification; healthcare information technology; interoperability; conformance; standards

\section{*Sheryl L. Taylor}

E-mail: sheryl.taylor@nist.gov 